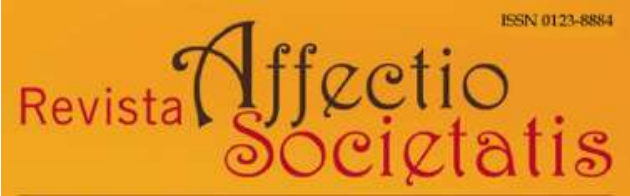

Departamento de Psicoanálisis | Universidad de Antioquia

Revista Affectio Societatis

Departamento de Psicoanálisis

Universidad de Antioquia

revistaaffectiosocietatis@udea.edu.co

ISSN (versión electrónica): 0123-8884

Colombia

2020

David Andrés Vargas Castro

Ataque de pánico y motivos de consulta en psicoanálisis

Revista Affectio Societatis, Vol. 17, N. ${ }^{\circ} 33$, julio-diciembre de 2020

Art. \# 3 (pp. 65-86)

Departamento de Psicoanálisis, Universidad de Antioquia

Medellín, Colombia 


\title{
ATAQUE DE PÁNICO Y MOTIVOS DE CONSULTA EN PSICOANÁLISIS ${ }^{1}$
}

\author{
David Andrés Vargas Castro ${ }^{2}$ \\ Universidad de la Marina Mercante \\ vargascastrod@yahoo.com.ar \\ https://orcid.org/0000-0002-7574-0586
}

DOI: 10.17533/udea.affs.v17n33a03

\section{Resumen}

En razón de una investigación que $D S M$, pasando luego a distinguir estamos realizando sobre los motivos de consulta en una institución psicoanalítica en la Ciudad Autónoma de Buenos Aires, el presente artículo tiene como propósito plantear la hipótesis de por qué el ataque de pánico se presenta como el motivo de consulta más frecuente. Para ello, iniciaremos con una descripción de la crisis de angustia freudiana y su derivación en el panic attack del entre ataque de pánico y otras presentaciones clínicas como fobia, síntoma conversivo y depresión. Finalmente, plantearemos que, en razón de las características del ataque de pánico, éste adviene como causa eficiente para demandar tratamiento.

Palabras claves: ataque de pánico, motivos de consulta, psicoanálisis, angustia señal, angustia traumática.

1 El presente artículo es resultado de la investigación recientemente concluida "Motivos de consulta de mayores de edad en institución de orientación psicoanalítica en la Ciudad Autónoma de Buenos Aires 2018-2020", financiada por la Universidad de la Marina Mercante.

2 Psicoanalista. Doctorando en Psicología (Universidad de Buenos Aires). Magister en Psicoanálisis (Universidad de Buenos Aires). Docente e investigador (Universidad de la Marina Mercante). Docente, investigador y analista del Servicio de Extensión Atención Clínica de Adultos (Universidad de Buenos Aires). Miembro del Foro Analítico del Río de la Plata. 


\section{PANIC ATTACK AND REASONS FOR CONSULTATION IN PSYCHOANALYSIS}

\begin{abstract}
Due to a research we are carrying out on the reasons for consultation in a psychoanalytic institution in the Autonomous City of Buenos Aires, this paper aims to raise the hypothesis of why the panic attack is the most frequent reason for consultation. To this end, we will begin with a description of the Freudian anxiety and its derivation in the DSM panic attack; then we distinguish between the panic attack
\end{abstract}

and other clinical presentations such as phobia, conversion symptoms, and depression. Finally, we will state that, due to the characteristics of the panic attack, this is an efficient cause to demand treatment.

Keywords: panic attack, reasons for consultations, psychoanalysis, anxiety as signal, traumatic anxiety.

\section{ATTAQUE DE PANIQUE ET RAISONS DE CONSULTATION EN PSYCHANALYSE}

\section{Résumé}

Cet article est issu d'une recherche sur les motifs de consultation dans une institution psychanalytique de la ville de Buenos Aires. L'objectif est donc d'émettre des hypothèses sur les raisons pour lesquelles les attaques de panique sont le motif de consultation le plus fréquent. Pour ce faire, nous commencerons par une description de la crise d'angoisse chez Freud et de sa classification en panic attack du DSM ; puis, nous distinguerons l'attaque de panique des autres présentations cliniques telles que la phobie, le symptôme de conversion et la dépression. Enfin, nous avancerons l'idée que, en raison des caractéristiques de l'attaque de panique, cette dernière est une raison valable pour demander un traitement.

Mots-clés : attaque de panique, motifs de consultation, psychanalyse, angoisse, signal, angoisse traumatique. 


\section{ATAQUE DE PÂNICO E MOTIVOS DE CONSULTA EM PSICANÁLISE}

\section{Resumo}

Devido a uma pesquisa que estamos a realizar sobre os motivos de consulta numa instituição psicanalítica da Cidade Autônoma de Buenos Aires, o presente artigo tem como objetivo propor a hipótese de por que o ataque de pânico é apresentado como o motivo mais frequente de consulta. Para isso, começaremos com uma descrição do ataque de angústia freudiano e sua derivação no panic attack do DSM; posteriormente, distingui- remos entre ataque de pânico e outras apresentações clínicas, como fobia, sintoma conversivo e depressão. Finalmente, propomos que, devido às características do ataque de pânico, este constitui uma causa eficiente para demandar tratamento.

Palavras-chave: ataque de pânico, motivos de consulta, psicanálise, angústia sinal, angústia traumática.

Recibido: 23/05/2019 • Aprobado: 16/02/2020 
Manos crispadas me confinan al exilio.

Ayúdame a no pedir ayuda. Me quieren anochecer, me van a morir. Ayúdame a no pedir ayuda.

Alejandra Pizarnik.

\section{Introducción}

En razón del avance de una investigación que venimos realizando a propósito de los motivos de consulta en una institución psicoanalítica en la Ciudad Autónoma de Buenos Aires, se nos plantea la pregunta con respecto al llamado "ataque de pánico" como motivo de consulta más frecuente.

Si bien el ataque de pánico es un significante relativamente nuevo en manuales internacionales de trastornos mentales, y otras perspectivas psicológicas han dedicado desarrollos al respecto, ya había sido descrito por Freud muy tempranamente en su obra. La bibliografía psicoanalítica se ha enfocado, principalmente, en el intento de pensar la nosografía descriptiva psiquiátrica a la luz de la explicación metapsicológica freudiana. Esfuerzo necesario y del cual el presente texto hará un esbozo, pero diferenciándose en el objetivo específico de articularlo con la eficacia que tiene el ataque de pánico para precipitar a los sujetos -casi de forma inmediata- a consultar, lo que lo distingue de otros motivos de consulta.

En razón de ello, iniciaremos haciendo una descripción de la investigación que funciona como motor de este texto. Seguidamente, realizaremos un breve recorrido desde la descripción realizada por Freud de la crisis de angustia a la consignada en el Manual Diagnóstico y Estadístico de los Trastornos Mentales (DSM) como "trastorno de pánico". Posteriormente, dedicaremos algunos apartados a diferenciar la angustia del síntoma conversivo, de la fobia, de las acciones obsesivas; indicar su relación con la depresión; establecer la diferencia freudiana entre angustia señal y angustia automática, así como la articulación entre desamparo y crisis de angustia. Finalmente, plantearemos los motivos de consulta articulados con la urgencia subjetiva y propon- 
dremos una hipótesis de por qué el ataque de pánico se presenta como causa eficiente de motivos de consulta.

\section{Consideraciones sobre nuestra investigación}

La investigación a la que nos encontramos abocados se inscribe en el campo del psicoanálisis de orientación lacaniana. Desde este marco teórico, la experiencia del análisis ha de ser entendida en la singularidad que atañe a cada sujeto, en su división subjetiva entre la pulsión y la forma en que se defiende de la pulsión, pero manteniendo una tensión con lo particular (Lombardi, 2009) que también lo constituye como sujeto en su relación al tipo clínico y al Otro al que pertenece con coordenadas sociales y políticas propias de cada época. Es también esta tensión entre lo particular y lo singular lo que permite la formalización de la experiencia del análisis, a saber, la clínica psicoanalítica, la exigencia que el analista deba dar cuenta de su práctica, de allí que Lacan (1974-1975) advierta que: “Es indispensable que el analista sea al menos dos. El analista para tener efectos y el analista que, a esos efectos, los teoriza" (Clase del 10 de diciembre de 1974).

Por otro lado, es menester destacar que, si bien el psicoanálisis distingue el motivo de consulta de la demanda de análisis (Lacan, 2008/1958), considera igualmente privilegiados los motivos de consulta, ya que gracias a ellos será posible dar lugar a las demandas de análisis, así como Freud (2003/1900) ubicó al contenido manifiesto del sueño como vía regia al inconsciente. De modo tal que la elección por parte del sujeto de presentar su malestar en un motivo de consulta específico, y de representarse en él, tiene su valor en sí mismo.

Dentro del amplio campo de la psicología, desde la perspectiva psicoanalítica, se han realizado pocas investigaciones de índole cuantitativa en torno a los motivos de consulta. Ubicaremos a continuación algunas de ellas.

Quesada (2004) agrupa dentro de los motivos de consulta más comunes los problemas sociales (elección vocacional, rendición de prue- 
bas, vínculos de amistad y de trabajo, empleo); problemas de pareja (celos, violencia); problemas familiares (relación padres e hijos, entre los padres, entre los hijos); problemas con la sexualidad (inhibiciones, disfunciones y dificultades en la elección de pareja); y síntomas somáticos (afecciones en el cuerpo que no se explican orgánicamente). Igualmente, la autora destaca las pocas investigaciones realizadas a propósito de los motivos de consulta, en contraste con las producciones en torno a los modos de tratamiento. Advierte, a su vez, que "resulta alarmante la escasez de datos que, por lo menos en nuestro país, se encuentra un investigador cuando sale a buscar experiencias que en un sentido similar se hayan realizado y formalizado", aclarando que "no creemos desde ya que las experiencias sean escasas, sino su transmisión" (Quesada, 2004, pág. 8).

Martínez (2006) distingue los motivos de consulta manifiestos de los latentes, siendo los primeros los que responden a la interpretación que hacen los pacientes de su malestar, mediatizados por sus experiencias, información, imaginario socio-cultural, etc.; y los segundos, los relativos a las percepciones subjetivas del paciente con respecto a su malestar. Igualmente, diferencia el motivo de consulta de la demanda de tratamiento, la cual implica un reconocimiento por parte del paciente de una necesidad subjetiva y un deseo de pedir ayuda.

En cuanto a estudios en el ámbito público, tal como el realizado por Sotelo, Belaga, Coronel, Leserre, y Jorge (2008), se ha logrado establecer que el $21 \%$ de las consultas se debe a problemas de consumo de sustancias, el $18 \%$ a trastornos de ansiedad, el $16 \%$ a trastornos depresivos, el $11 \%$ a trastornos delirantes, y un $10 \%$ a los otros pedidos de consulta menores.

Haldemann y Muraro (2009), en el marco de la investigación UBACyT, "Momentos electivos en el tratamiento psicoanalítico de las neurosis en el servicio de Clínica de Adultos de la Facultad de Psicología, UBA", señalan que un $48 \%$ de los motivos de consulta concierne al afecto de tristeza -"me siento deprimido", "no tengo ganas de hacer nada"-; siendo más habitual el que se apuntala en expresiones en torno a la angustia. 
En este mismo marco, Muraro y Gurevich (2011) diferencian entre el motivo de consulta y la causa eficiente, es decir, la razón que manifiesta el paciente de por qué consulta y qué hizo que consultara ahora y no en otro momento, localizando así dos grupos: el primero, pacientes que han sostenido durante mucho tiempo un padecimiento y que, en un momento determinado, se han sentido sobrepasados; y el segundo grupo de consultantes, quienes en razón de un acontecimiento abrupto se han precipitado a la consulta.

Teniendo en cuenta estos antecedentes, el objetivo general de nuestra investigación consiste en identificar, caracterizar y analizar los motivos de consulta en personas mayores de edad que concurren con propósito de tratamiento a una institución de orientación psicoanalítica en la Ciudad Autónoma de Buenos Aires en el período 2018-2020.

Para alcanzar tal objetivo, los pasos que seguiremos serán los siguientes: a) Organizar las entrevistas de admisión de los pacientes que concurren a la institución psicoterapéutica elegida desde los aspectos sociológicos, tales como edad, sexo, ocupación, estado civil, escolaridad, grupo familiar, lugar de residencia; b) Caracterizar a los sujetos acorde a sus motivos de consulta; c) Analizar los motivos de consulta a la luz del marco teórico planteado; d) Realizar entrevistas a psicoanalistas que llevan a cabo las entrevistas de admisión para extraer sus impresiones sobre los motivos de consulta en el marco de la institución psicoterapéutica elegida; e) Comparar los resultados de los motivos de consulta con los datos otorgados por los psicoterapeutas; f) Extraer conclusiones posibles y alternativas de futuras investigaciones.

En cuanto a la metodología, se llevará a cabo una investigación empírica cuantitativa y cualitativa de tipo exploratorio-descriptivo, de corte transversal, inter-sujetos. La población estudiada serán los sujetos mayores de edad que pidan consulta psicológica en una institución psicoterapéutica de orientación psicoanalítica en la Ciudad Autónoma de Buenos Aires. Las variables sociológicas a tener en cuenta serán: sexo, grupo familiar, escolaridad, ocupación, estado civil. El instrumento a usarse será el protocolo concerniente a la entrevista de recepción como material privilegiado (Haldermann y 
Muraro, 2009) que ya tiene confeccionada la institución psicoterapéutica elegida, dentro de la cual encontramos las variantes antes señaladas. Igualmente, se hará uso del programa Atlas.ti para análisis de datos cualitativos y cuantitativos. Posteriormente, haciendo uso del marco teórico psicoanalítico, se realizará un análisis de los datos recabados.

Si bien la investigación aún se encuentra en curso, dentro de los resultados parciales, y tal como lo advertimos en nuestra introducción, podemos ubicar al ataque de pánico como el mayor motivo de consulta localizado por los terapeutas (70 \%).

\section{De la crisis de angustia al ataque de pánico}

En el intento del DSM por proponerse como ateórico, las clasificaciones de "neurosis" fueron eliminadas de éste, siendo sustituidas por los trastornos de ansiedad. Dentro del apartado dedicado a dichos trastornos, podemos encontrar al ataque de pánico (panic attack).

Según la quinta versión del DMS (2014, págs. 133-134), hallamos la siguiente definición del ataque de pánico, así como la enumeración de sus síntomas para establecer su diagnóstico:

Un ataque de pánico es la aparición súbita de miedo intenso o de malestar intenso que alcanza su máxima expresión en minutos y durante este tiempo se producen cuatro (o más) de los síntomas siguientes:

1. Palpitaciones, golpeteo del corazón o aceleración de la frecuencia cardíaca.

2. Sudoración.

3. Temblor o sacudidas.

4. Sensación de dificultad para respirar o de asfixia.

5. Sensación de ahogo.

6. Dolor o molestias en el tórax. 
7. Náuseas o malestar abdominal.

8. Sensación de mareo, inestabilidad, aturdimiento o desmayo.

9. Escalofríos o sensación de calor.

10. Parestesias (sensación de entumecimiento o de hormigueos).

11. Desrealización (sensación de irrealidad) o despersonalización (separarse de uno mismo).

12. Miedo a perder el control o de "volverse loco".

13. Miedo a morir.

Como lo señala Quesada (2010), si bien no es posible afirmar que haya algo novedoso en la caracterización del trastorno que Freud ya había descrito tiempo atrás -y como veremos a continuación-, sí es nueva la gran frecuencia como motivo de consulta en consultorios privados o guardias hospitalarias, lo que nos puede hacer pensar que algo de la época propicia esta forma de manifestación de angustia. No hay que perder de vista el lugar central que en nuestra época tiene la Internet y los buscadores de información, de allí que, luego de un episodio de angustia, antes de dirigirse a un profesional de la salud mental, los consultantes suelen realizar una búsqueda de los síntomas que han tenido, presentándose a la consulta diciendo que tuvieron un ataque de pánico. Será necesario, escucha mediante, tomar esta presentación como un significante en el que el sujeto se representa, sin definición diagnóstica alguna. Es así que se hace necesario no comprender, como lo recomienda Lacan al psicoanalista, para dar lugar a que el sujeto pueda desplegar su padecimiento.

En "Sobre la justificación de separar de la neurastenia un determinado síndrome en calidad de 'neurosis de angustia'", encontramos la "crisis de angustia" con las características planteadas en el DSM. El creador del psicoanálisis la enmarcó dentro de las neurosis de angustia y la diferenció de las neurosis actuales. He aquí la descripción realizada en dicho texto freudiano:

Un ataque tal puede consistir en el sentimiento de angustia solo, sin ninguna representación asociada, o bien mezclarse con la interpretación más espontánea, como la aniquilación de la vida, 
"caer fulminado por un síncope", la amenaza de volverse loco; o bien el sentimiento de angustia se contamina con una parestesia cualquiera (semejante al aura histérica), o, por último, se conecta con la sensación de angustia una perturbación de una o varias funciones corporales -la respiración, la actividad cardiaca, la inervación vasomotriz, la actividad glandular-. De esta combinación, el paciente destaca ora un factor, ora el otro: se queja de "espasmos en el corazón", "falta de aire", "oleadas de sudor", "hambre insaciable", etc., y en su exposición es frecuente que el sentimiento de angustia quede completamente relegado o se vuelva apenas reconocible como un "sentirse mal", un "malestar" (...) Según esto, existen ataques de angustia rudimentarios y equivalentes del ataque de angustia [énfasis agregado], probablemente de igual significado, que muestran una gran riqueza de formas, poco apreciadas hasta ahora. El estudio más preciso de estos estados de angustia larvada y su distingo diagnóstico de otros ataques es una tarea que los neuropatólogos deberían abordar con urgencia. (Freud, 2003/1895, págs. 94-95).

Como es notoria, esta extensa cita no sólo muestra la similitud, punto por punto, con las características consignadas en el DSM-5, sino que Freud ubica la importancia de la distinción diagnóstica que caracteriza como urgente.

Se hace evidente, entonces, que aquello que el DSM sacó por la ventana, ha retornado por la puerta. A propósito de esto, estamos de acuerdo con Baumgart (2010) en que el ataque de pánico se ha convertido en el síntoma de la psiquiatría contemporánea y que, como todo síntoma, está constituido en dos tiempos, siendo el primer tiempo olvidado -nosotros preferiremos decir reprimido, denotando el esfuerzo de desalojo del cual es presa-, por lo que retorna como novedad.

Para continuar con nuestra exposición, realicemos un breve resumen de los planteamientos efectuados por Freud en "Inhibición, síntoma y angustia", en el que reformula sus postulados sobre la relación entre angustia y represión.

Previamente a este texto, Freud ubica a la angustia como consecuencia de la represión al determinar que dicho afecto era resultado 
de una libido no ligada que no encontraba vía de descarga, por lo cual se trasmudaba en angustia. Los destinos de esta libido no tramitada se manifestaban en los diversos síntomas de las neurosis de angustia: histeria, obsesión, fobia. Luego, gracias a su texto "Más allá del principio del placer", Freud advierte que el aparato psíquico no sólo funciona bajo el primado del principio del placer, sino que se presentan manifestaciones psíquicas que contrarían ese principio, como las neurosis traumáticas, los fenómenos de repetición en la cura y algunos juegos infantiles.

Estas formulaciones harán que en "Inhibición, síntoma y angustia" ubique a la angustia como previa a la represión, además de diferenciar dos modalidades de la angustia: angustia señal y angustia traumática. La primera tiene lugar en el yo como almácigo de la angustia, teniendo como objetivo advertir al aparato psíquico de una situación en la que puede sufrir un trauma. De esta forma, al modo de una vacuna, sufre la angustia como un anticipo que lo prepara para el ataque o la huida. La segunda implica una ruptura de las barreras de contacto y de las defensas, en tanto ocurre algo que -ya sea en el interior o en el exterior- lo deja indefenso, desamparado del recurso de la palabra, por su carácter disruptivo, inesperado, superando la posibilidad de tramitar tan alto monto de excitación. Precisamente, Freud adjetiva como "traumática" a esta modalidad de la angustia porque produce un agujero en la barrera antiestímulos, perdiendo el sujeto la posibilidad de prepararse para hacerle frente gracias a su naturaleza inesperada.

Esta diferenciación ya estaba esbozada en las elaboraciones de "Más allá del principio del placer", tal como podemos leer a continuación:

Descubrimos, así, que el apronte angustiado, con su sobreinvestidura de los sistemas recipientes, constituye la última trinchera de la protección antiestímulo. En toda una serie de traumas, el factor decisivo para el desenlace quizá sea la diferencia entre los sistemas no preparados y los preparados por sobreinvestidura. (Freud, 2003/1920, pág. 31). 


\section{Distinciones clínicas: conversión histérica, acciones} obsesivas, fobia, depresión

Haciendo eco del carácter de urgencia con el que Freud considera que debe distinguirse el diagnóstico entre la crisis de angustia de otras manifestaciones de dicho afecto, proseguiremos a continuación.

En la nosografía freudiana podemos encontrar, dentro del éxito por parte del aparato psíquico de tramitar la angustia, una oposición entre histeria de conversación y ataque de pánico, basada en que el síntoma conversivo es el más exitoso porque evita la aparición de angustia, contrario al ataque de pánico que da cuenta de una dificultad del aparato psíquico de ligar ese afecto a alguna representación, de allí que no podamos hablar de un significado reprimido, presto a interpretación, como ocurre en el caso de la conversión.

Es de destacar que, si bien en nuestra investigación el ataque de pánico aparece como el mayor motivo de consulta, el menos frecuente son las fobias. A nuestro criterio, no es casualidad que se produzca esta oposición. Con respecto a la fobia, frente a la angustia, al estar circunscrito en la mayoría de los casos el objeto fobígeno, las conductas evitativas son exitosas, o el objeto contrafóbico cumple su función defensiva. El caso Hans lo muestra claramente al poner en evidencia la expresión de angustia generalizada que encuentra su delimitación en la fobia al caballo, aunque luego expandiéndose a otros objetos de forma metonímica relacionados al caballo, como el carruaje. Es así que la fobia implica la participación de la represión, el desplazamiento de la representación. Por el contrario, la crisis de angustia "no engaña", siguiendo la afirmación de Lacan (2006/19621963) en torno a la angustia: no hay allí significantes a interpretar. El trabajo analítico al respecto concierne en ligar otro significante para que el sujeto pueda representarse, so pena de quedar en el lugar de objeto. No por casualidad Lacan (2006/1975-1976) liga a la angustia con la sensación de quedar reducidos a un cuerpo.

Fruto de este trabajo de ligar la angustia a alguna representación, encontramos que, luego del primer episodio de un ataque de pánico, 
y en el intento del sujeto por encontrar una causa de lo sucedido, se emprendan en algunas ocasiones conductas evitativas. Por ejemplo, si se produjo en un autobús, se evita hacer uso de dicho medio de transporte. Hallamos así, a pesar de sus diferencias, similitudes entre la fobia y el ataque de pánico. Sin embargo, y siguiendo la relación entre los motivos de consulta por ataque de pánico como el más frecuente y la fobia como el menos frecuente, queda evidenciado por la oposición que implican entre objetivar y no poder objetivar la fuente de angustia.

Con respecto al ritual obsesivo, estos tienen una naturaleza compulsiva, en tanto que, si bien el paciente sabe que ha realizado alguna acción, vía la duda, nuevamente la realiza, so pena de irrumpir la angustia. Igualmente, es notable cómo estos rituales suelen ser expansivos -punto en común con lo señalado más arriba-, sucediendo entonces que, inexorablemente, no pueden ser realizados, teniendo lugar así la angustia.

Por otro lado, si bien la angustia y la llamada depresión presentan una fenomenología sumamente distinta, es de destacar que, tal como lo señala Quesada (2010), "estudios desde la psicología cognitiva, especialmente los de Aaron Beck y Arthur Freeman (1990), han encontrado comorbilidad del trastorno de pánico con depresión mayor, lo cual conduce a asociar este trastorno con riesgo suicida" (pág. 9).

De esta forma aparece anudada la depresión con la angustia, pero siendo más eficaz el ataque de pánico para precipitar a consultar que la depresión, debido a que, en la mayoría de los casos, el paciente es derivado por el trabajo o por algún familiar, en razón de que las manifestaciones sintomáticas son más introvertidas que las del ataque de pánico, así como las primeras suelen ser más del orden crónico, mientras que las segundas son agudas. De hecho, es importante señalar que la depresión suele también presentarse como cansancio extremo resultante de trabajo o de problemas familiares, siendo necesario un trabajo minucioso para realizar un diagnóstico diferencial; contrario a la presentación del ataque de pánico en donde el paciente, en un primer momento, no logra asociarlo a alguna problemática precedente. 
En términos lacanianos, si bien el fantasma es la guarida del sujeto frente al deseo del Otro, guarida que, al modo de la Casa tomada cortasiana, puede ir asfixiándolo al suponer poder responder al objeto del deseo con el objeto de la demanda, es, al fin y al cabo, una angustia enmarcada -como el fantasma mismo lo es-. La angustia automática o traumática está en solidaridad, por el contrario, con el fracaso del losange como velo del sujeto al objeto en el fantasma (Amigo, 2007), allí donde lo sorpresivo irrumpe y lo desaloja de su posición de sujeto de la palabra.

No finalicemos este apartado sin tener en cuenta lo señalado por Lacan en "De nuestros antecedentes" a propósito del fenómeno de la despersonalización, en donde es claro al advertir que no es un dato diagnóstico, ya que puede responder a diversos tipos clínicos. Advertencia que nos recuerda que el diagnóstico en psicoanálisis es en transferencia y no determinado por síntomas:

Para los puntos de referencia del conocimiento especular finalmente recordamos una semiología que va desde la más sutil despersonalización hasta la alucinación del doble. Se sabe que no tienen en sí mismos ningún valor diagnóstico en cuanto a la estructura del sujeto (la psicótica entre otras). (Lacan, 2010/1966, pág. 78).

Es así como no es lo mismo la despersonalización en el marco de una psicosis -como puede darse en la esquizofrenia y los fenómenos de cuerpo fragmentado- que en el campo de la neurosis -en las manifestaciones de un ataque de pánico-.

\section{¿Angustia o pánico?}

Entre los autores que han trabajado el ataque de pánico desde el psicoanálisis podemos ubicar dos posturas: una, en la que se considera al ataque de pánico como concerniente a una crisis de angustia; y otra, en la que se afirma que se trata de pánico; estas dos posturas responden a momentos distintos de los desarrollos freudianos. Ya nos ocupamos previamente de lo que Freud delimita como crisis de angustia. Con respecto al pánico, el creador del psicoanálisis hará referencia al 
respecto en "Psicología de las masas y análisis del yo", diciendo que es el resultado de la descomposición de la masa, al perderse la doble ligazón libidinal, a saber, la referida al líder y a los otros individuos de la masa: "Lo caracteriza el hecho de que ya no se presta oídos a orden alguna del jefe, y cada uno cuida por sí sin miramiento por los otros. Los lazos recíprocos han cesado, y se libera una angustia enorme, sin sentido" (Freud, 2003/1921, pág. 91). Es precisamente esta angustia enorme carente de sentido la que ha sido considerada como equivalente al ataque de pánico. Si tenemos en cuenta el título del texto, advirtiendo que tanto la masa como el yo comparten la misma psicología -o, más precisamente, la misma metapsicología-, podemos pensarlo en el orden de cierta descomposición del yo, o por lo menos, una suspensión a la función de desconocimiento propia del yo (Lacan, 2010/1949) al ponerse en evidencia su dimensión de objeto. El fenómeno de despersonalización del ataque de pánico da cuenta de ello si recordamos al yo en términos de cuerpo y superficie. Si el yo está alienado a la imagen del otro, no es sin la intervención del Otro como soporte de esa imagen, de modo tal que la suspensión de la función del Otro traiga consigo dicho efecto en el que el sujeto se advierte como objeto. No por casualidad, y en relación al temor a volverse loco, uno de los ejemplos que da Freud de pánico concierne a que “¡el general ha perdido la cabeza!" (Freud, 2003/1921, pág. 93), lo que produce la descomposición de la masa.

A nuestro entender, no se trata de decidir si se trata de angustia o pánico, sino de pensarlos articulados en el ataque de pánico ya que, si bien la crisis propiamente dicha responde a las coordenadas subjetivas del pánico, luego del primer episodio, y tal como lo señalamos previamente, en la mayoría de los casos el sujeto queda en expectativa angustiante, en alerta, lo que responde a la angustia como señal, produciéndose, a su vez, el fenómeno ampliamente descrito por diversas perspectivas, a saber, un efecto de retroalimentación en el que la expectativa angustiante de tener una crisis de angustia genera una crisis de angustia.

Dentro de los estudios más representativos sobre el ataque de pánico desde el psicoanálisis, Quesada (2010) considera que no se realiza una tramitación psíquica, sino que directamente el monto de 
excitación se deriva al cuerpo, no al modo de la histeria, donde es la representación la que sufre tal destino, sino al cuerpo en su dimensión más real, siendo la idea de volverse loco o de morir una ligazón a esa inervación desbordada, por más insoportable que sea. Distingue así entre pánico y angustia, sirviéndose de las elaboraciones de Freud en "Más allá del principio del placer".

Sin embargo, y a diferencia de lo señalado por la autora, consideramos que es necesario advertir que con las teorizaciones de "Inhibición, síntoma y angustia", lo que Freud llamó pánico queda absorbido en lo que desarrolla como angustia traumática en razón de su naturaleza sorpresiva y sin objeto aparente que la desencadene. Decimos "aparente" puesto que con los desarrollos lacanianos se formaliza el objeto en cuestión -el objeto $a$ - como un objeto no especularizable y, por tanto, sin remisión a ningún objeto fenoménico, sin ningún significante que pueda ubicarse en el campo del Otro.

\section{Desamparo y crisis de angustia}

Como vimos, en la angustia automática el sujeto se encuentra desamparado del recurso de la palabra, lo que remite al desamparo en términos del Otro primordial. Freud (2003/1950) plantea que, en razón de lo prematuro en que nace el ser humano, se requiere la intervención de un Otro auxiliar, el cual puede suponer allí o no un sujeto. Por ejemplo, si un niño llora, se puede suponer que tiene frío, hambre, quiere mimos, etc. De allí que Freud hable de "acción específica" como aquella con la que el Otro auxiliar reduce la excitación. Esta acción específica no debe confundirse con una especificidad a nivel de correlación entre un objeto y una acción. Es una operación de lectura, una interpretación, siendo el Otro el primer intérprete, dando palabras y, por ende, sentido, a lo ocurrido. Vemos que, en la crisis de pánico, precisamente, esta función se encuentra suspendida.

A propósito de la relación entre desamparo y crisis de angustia, Marchesini (2008) ubica ciertas particularidades de nuestra época que son solidarias a dicha relación, como la descomposición del tejido so- 
cial y la sensación de soledad, destacando, igualmente, la imposibilidad de generalizar una explicación al respecto si se pretende una elucidación sociológica, puesto que no todos los sujetos responden de la misma manera a las vicisitudes de la época:

Asistimos a distintos sucesos de la época: inseguridad social, incertidumbre, robos, presiones de la vida moderna, inestabilidad económica, un contexto social que se descompone fácilmente, sensación de soledad. La experiencia de la angustia insiste y resiste en el sujeto de nuestro tiempo. Se vive en un estado de ansiedad generalizada, en alerta, bajo el eterno retorno de algo inquietante. Pero en este estado de cosas no todos sucumben al pánico, y es por ello lícito considerar este fenómeno, sin precedentes en la vida de alguien, como un síntoma de la vida privada. Si bien las culturas producen sus síntomas, éstos se construyen de modo singular en los sujetos. (23 de agosto de 2008).

\section{Los motivos de consulta y la urgencia subjetiva}

Es bien conocido que antes de consultar con un analista los sujetos suelen consultar a otros profesionales. En el caso del ataque de pánico con mayor frecuencia, por la cascada de reacciones que desencadena en el cuerpo, como pudimos ver previamente.

Lo que en psicoanálisis conocemos como "urgencia subjetiva" no es equivalente a la urgencia médica, al no tratarse de un asunto de vida o muerte. Sin embargo, es gracias a que puede ubicarse una demanda de análisis en términos de urgencia que un análisis encuentra su empuje. Por esta razón, Lacan (2012/1977) planteó al análisis en términos de urgencia. Contraria a la idea del psicoanálisis interminable, la idea de urgencia responde a que algo empuje al trabajo analizante para desembarazarse del síntoma. Es así que un motivo de consulta no es necesariamente una demanda de análisis, pero nos parece fundamental ubicar el valor del motivo de consulta, siguiendo la metáfora freudiana, como la arenilla -demanda de verdad, que podemos hacer equivalente con el motivo de consulta- de la que se forma la perla -verdadera demanda, o demanda de análisis-. 
El establecimiento de la urgencia subjetiva sólo se da en transferencia, al ubicar el síntoma analítico. Así como vimos con la urgencia, no se solapa con el síntoma médico, sino que se aísla como aquello posible de ser analizado. De allí que sea fundamental que el analista, vía el manejo de la transferencia, se abstenga de responder a una demanda que se recubre -especialmente en el ataque de pánico- en un pedido de auxilio. La escucha analítica tiene, en estos casos, la función de red para que allí pueda el sujeto volver a representarse en los significantes, lo que es, nuevamente, dirigirse al Otro en tanto lugar de la palabra, dar un lugar en el que la pulsación del inconsciente encuentre la temporalidad que abra al tiempo de comprender, otro tiempo distinto al del instante subjetivamente interminable del ataque de pánico, en el que sea posible historizar y dar relato a eso que se presenta como inefable.

\section{Las características del ataque de pánico: ¿causa eficiente?}

Nos resulta igualmente interesante que dentro de los motivos de consulta que normalmente continúan un tratamiento hasta su culminación en el tiempo institucional, y en algunos casos, interesados en proseguir su análisis en consultorio privado, el ataque de pánico sea igualmente el más frecuente. De allí que, siguiendo a Muraro y Gurevich (2011), nos preguntemos si el ataque de pánico funciona como causa eficiente, entendiendo esto como lo decisivo en precipitar al paciente a la consulta.

A diferencia del síntoma, con el que el sujeto suele arreglárselas -lejos de la idea de que sólo al final del análisis lo logra-, que en un principio es goce y no llama a la interpretación (Lacan, 2006/19621963), y hasta sirve al yo para afirmarse y obtener ganancia del mismo -lo que aparece en Freud (2003/1926) como ganancia secundaria-, el ataque de pánico suele precipitar inmediatamente a la consulta. Luego del primer episodio, con frecuencia, se produce el fenómeno de la angustia como señal, como una alerta de la que no se sabe de qué se trata. Creemos que en esta dirección es que el ataque de pánico es solidario de la demanda de verdad, en tanto que, desde el principio, se presenta como egodistónico, no asimilable por el yo. 
Es de destacar que el ataque de pánico, cuando no remite al psiquiatra, es por los prejuicios que se tienen en torno a la toma de medicación, como es que se restrinja sólo a los "locos" o que producirá efectos secundarios indeseados, como obesidad y adicción. Esto queda evidenciado cuando, en el transcurso de los tratamientos, se sugiere interconsulta psiquiátrica, ya que varios pacientes presentan reticencia al respecto.

Lo crucial en juego es que no sólo el sujeto llegue a la consulta, sino que sea posible un tratamiento analítico. Como ya lo señalaba Freud, preservar el sufrimiento es también necesario para que el análisis no se reduzca a una terapéutica. De allí que el movimiento sea pasar del ataque de pánico a la constitución de un síntoma analítico, el cual hace del analista un objeto traumatizante vía la neurosis de transferencia. Este movimiento posibilita motorizar la cura al ubicar una causa subjetiva del sufrimiento y, al mismo tiempo, no dejar al sujeto en el desamparo del silencio del pánico. No es de extrañar que en el relato de los pacientes -y también como adelanto de nuestra investigación- se pueda localizar una pérdida inesperada, ya sea de trabajo o de amor, los dos ejes libidinales principales del sujeto.

Finalmente, estamos de acuerdo con Cosaka (2010) en ubicar al ataque de pánico como paradigma de la posmodernidad, al dar cuenta de la fragmentación y endeblez de los lazos sociales que el "discurso" capitalista - precisamente por ser un falso discurso- promueve.

\section{Conclusiones}

Vimos la equivalencia, punto por punto, entre lo descrito por Freud como crisis de angustia y lo que posteriormente aparecerá en el DSM como ataque de pánico, destacando el carácter de urgencia con la que el psicoanalista vienés caracterizó a la distinción diagnóstica entre esta presentación subjetiva y otras presentaciones de angustia. Igualmente, hicimos un esbozo de los desarrollos sobre la angustia como resultante de la represión, así mismo de la angustia como causa de la represión, giro producido en "Inhibición, síntoma y angustia" que 
dio lugar a las dos modalidades de la angustia, como señal y traumática, así como señalamos que ya previamente en "Más allá del principio del placer" se encontraba trazada esta diferencia.

Hicimos la distinción clínica entre ataque de pánico y otras presentaciones subjetivas como conversión histérica, fobia, compulsiones obsesivas, depresión, en relación a la angustia y al objeto, ubicando allí que la histeria es la forma más exitosa con respecto a la tramitación de la angustia en oposición a la crisis de angustia. Igualmente, advertimos que el fenómeno de despersonalización no es un dato diagnóstico.

Destacamos dos posturas psicoanalíticas con respecto al ataque de pánico: una que la considera como crisis de angustia y otra como pánico. Planteamos que, lejos de una oposición, era necesario pensar la usual respuesta subjetiva posterior al primer episodio de pánico, al producirse una angustia a modo de señal que desencadena nuevos episodios.

Señalamos la relación entre ataque de pánico y desamparo, entendiendo el desamparo como remitiendo a la ausencia de interpretación por parte del Otro, por lo que se ve truncada la tramitación simbólica de la irrupción de lo real, estando esto agudizado por la actual descomposición del tejido social.

Establecimos cómo los motivos de consulta en sí mismos no son suficientes para posibilitar un tratamiento, siendo necesaria la ubicación del síntoma como motor del análisis al rectificar al sujeto con respecto a su padecimiento, necesidad que le da al análisis su carácter de urgencia.

Planteamos la hipótesis de que el ataque de pánico resulta como causa eficiente por la necesidad de acudir a un Otro que viabilice un $\mathrm{S}_{2}$, un segundo significante que permita anudar esa angustia a alguna representación, posibilitando así la cura por la palabra.

Queda para posteriores artículos abocarnos a otros resultados que la investigación está por brindarnos, como las diferencias y simi- 
litudes en los motivos de consulta de hombres y mujeres, el rango de edad que consulta con mayor y menor frecuencia, la relación entre los motivos de consulta y el sostenimiento del tratamiento.

\section{Referencias}

Álvarez, C. (2003, agosto). El ataque de pánico, una mirada psicoanalítica. Imago agenda. http://www.imagoagenda.com/articulo.asp?idarticulo=753.

Asociación Americana de Psiquiatría. (2014). Guía de consulta de los criterios diagnósticos del DSM 5. Asociación Americana de Psiquiatría.

Amigo, S. (2007). Clínica de los fracasos del fantasma. Letra Viva.

Baumgart, A. (2010). Ataque de pánico y subjetividad. Eudeba.

Cosaka, J. (2010). Ataque de pánico. Una lectura psicoanalítica. Letra Viva.

Freud, S. (2003/1895). Sobre la justificación de separar de la neurastenia un determinado síndrome en calidad de "neurosis de angustia". En J. Strachey (Ed.) y J.L. Etcheverry y L. Wolfson (Trads.), Obras completas (Vol. III, págs. 85-115). Amorrortu.

Freud, S. (2003/1900). La interpretación de los sueños. En J. Strachey (Ed.) y J.L. Etcheverry y L. Wolfson (Trads.), Obras completas (Vols. IV-V). Amorrortu.

Freud, S. (2003/1909). Análisis de la fobia de un niño de cinco años. En J. Strachey (Ed.) y J.L. Etcheverry y L. Wolfson (Trads.), Obras completas (Vol. X, págs. 1-118). Amorrortu.

Freud, S. (2003/1909). A propósito de un caso de neurosis obsesiva. En J. Strachey (Ed.) y J.L. Etcheverry y L. Wolfson (Trads.), Obras completas (Vol. X, págs. 119-194). Amorrortu.

Freud, S. (2003/1920). Más allá del principio del placer. En J. Strachey (Ed.) y J.L. Etcheverry y L. Wolfson (Trads.), Obras completas (Vol. XVIII, págs. 1-62). Amorrortu.

Freud, S. (2003/1921). Psicología de las masas y análisis del yo. En J. Strachey (Ed.) y J.L. Etcheverry y L. Wolfson (Trads.), Obras completas (Vol. XVIII, págs. 63-136). Amorrortu.

Freud, S. (2003/1926). Inhibición, síntoma y angustia. En J. Strachey (Ed.) y J.L. Etcheverry y L. Wolfson (Trads.), Obras completas (Vol. XX, págs. 71-161). Amorrortu.

Freud, S. (2003/1950). Proyecto de psicología. En J. Strachey (Ed.) y J.L. Etcheverry y L. Wolfson (Trads.), Obras completas (Vol. I, págs. 323-436). Amorrortu.

Haldermann, G. y Muraro, V. (2009). Algunas conclusiones inesperadas. En G. Lombardi (Comp.), Singular, particular, singular (págs. 173-179). JVE. 
Lacan, J. (1974-1975). Seminario R.S.I. Escuela Freudiana de Buenos Aires. Inédito. Lacan, J. (2012/1977). Prefacio a la edición inglesa del Seminario 11. En Esperanza, G. y otros (Trads.). Otros escritos (págs. 599-602). Paidós.

Lacan, J. (2006/1962-1963). El seminario. Libro 10: La angustia. Paidós.

Lacan, J. (2006/1975-1976). El Seminario de Jacques Lacan. Libro 23: El sinthome. Paidós.

Lacan, J. (2008/1958). La dirección de la cura y los principios de su poder. En T. Segovia (Trad.), Escritos (Vol. 2, págs. 565-626). Siglo Veintiuno Editores.

Lacan, J. (2010/1949). El estadio del espejo como formador de la función del yo (je) tal y como se nos revela en la experiencia psicoanalítica. En T. Segovia (Trad.), Escritos (Vol. 1, págs. 99-105). Siglo Veintiuno Editores. Lacan, J. (2010/1966). De nuestros antecedentes. En T. Segovia (Trad.), Escritos (Vol. 1, págs. 73-79). Siglo Veintiuno Editores.

Lombardi, G. (2009). Singular, particular, singular: La función del tipo clínico en psicoanálisis. En G. Lombardi (Comp.), Singular, particular, singular (págs. 17-23). JVE.

Lunger, V. (2013, 25 de marzo). Pánico. El sigma. Recuperado de http://www. elsigma.com/introduccion-al-psicoanalisis/panico/12545.

Marchesini, A. (23 de agosto de 2008). El ataque de pánico. Rioja virtual. http:/www.eol.org.ar/template.asp?Sec=prensa\&SubSec=america\&Fil e=america/2008/08_08_23_marchesini_ataque.html.

Martínez, P. (2006). Del motivo de consulta a la demanda en psicología. Revista de la Asociación Española de Neuropsiquiatría, 26(97), 53-69.

Muraro, V. y Gurevich, M. (2011). La causa eficiente. Memorias III Congreso Internacional de Investigación y Práctica Profesional en Psicología XVIII. Jornadas de Investigación. Séptimo Encuentro de Investigadores en Psicología del Mercosur (págs. 101-105). Facultad de Psicología, Universidad de Buenos Aires, Argentina.

Quesada, S. (2004). Estudio sobre los motivos de consulta psicológica en una población universitaria. Universitas Psychologica, 3(1), 7-16.

Quesada, S. (2010). Una explicación psicoanalítica del ataque de pánico. Letra Viva.

Sotelo, I., Belaga, G., Coronel, M., Leserre, L. y Jorge, J. (2008). Análisis de la demanda e intervenciones en la urgencia. Investigación en el Hospital Central de San Isidro. Investigaciones en Psicología, 2(13), 117-138. 